\title{
Aspheric Solute lons Modulate Gold Nanoparticle Interactions in an Aqueous Solution: An Optimal Way to Reversibly Concentrate Functionalized Nanoparticles
}

\author{
Oscar D Villarreal ${ }^{\mathrm{a}}$, Liao Y Chen ${ }^{\mathrm{a}, \mathrm{b}}$, Robert L Whetten ${ }^{\mathrm{a}}$, and Borries Demeler ${ }^{\mathrm{c}}$ \\ aDepartment of Physics and Astronomy, University of Texas at San Antonio, One UTSA Circle, \\ San Antonio, TX 78249 \\ 'Department of Biochemistry, The University of Texas Health Science Center at San Antonio, \\ San Antonio, TX 78229
}

\section{Abstract}

\begin{abstract}
Nanometer-sized gold particles (AuNPs) are of peculiar interest because their behaviors in an aqueous solution are sensitive to changes in environmental factors including the size and shape of the solute ions. In order to determine these important characteristics, we performed all-atom molecular dynamics simulations on the icosahedral $\mathrm{Au}_{144}$ nanoparticles each coated with a homogeneous set of 60 thiolates (4-mercapto-benzoate, pMBA) in eight aqueous solutions having ions of varying sizes and shapes $\left(\mathrm{Na}^{+}, \mathrm{K}^{+}\right.$, tetramethylamonium cation $\mathrm{TMA}^{+}$, trisamonium cation $\mathrm{TRS}^{+}, \mathrm{Cl}^{-}$, and $\mathrm{OH}^{-}$). For each solution, we computed the reversible work (potential of mean of force) to bring two nanoparticles together as a function of their separation distance. We found that the behavior of pMBA protected $\mathrm{Au}_{144}$ nanoparticles can be readily modulated by tuning their aqueous environmental factors ( $\mathrm{pH}$ and solute ion combinations). We examined the atomistic details on how the sizes and shapes of solute ions quantitatively factor in the definitive characteristics of nanoparticle-environment and nanoparticle-nanoparticle interactions. We predict that tuning the concentrations of non-spherical composite ions such as $\mathrm{TRS}^{+}$in an aqueous solution of AuNPs be an effective means to modulate the aggregation propensity desired in biomedical and other applications of small charged nanoparticles.
\end{abstract}

\section{INTRODUCTION}

Intensive research of functionalized gold nanoparticles (AuNPs) has been motivated by the promising applicability in chemical and biomedical procedures. ${ }^{1}$ Their characteristics in aqueous solutions have been investigated in both in vitro/vivo ${ }^{2-12}$ and in silico ${ }^{13-21}$ experiments. AuNPs interact with, for example, amyloids, ${ }^{22}$ viruses, ${ }^{23}$ bacteria, ${ }^{24}$ proteins, ${ }^{25-26}$ and DNA. ${ }^{27} \mathrm{~A}$ key issue in the fundamental understanding and the practical

\footnotetext{
${ }^{\mathrm{b}}$ Correspondence to Liao Y Chen, Liao.Chen@utsa.edu.. SUPPORTING INFORMATION

In the supporting information (SI) available online, we give all the details of the procedures to build the model systems, to run the equilibrium molecular dynamics (MD), to run the nonequilibrium steered molecular dynamics (SMD), the work curves from the SMD runs, the formulas to compute the PMF from SMD work data, and the formulas to estimate the aggregation propensity from the PMF curves.
} 
applications is how AuNPs interact with one another in an aqueous solution. Depending on the intended application, the AuNP propensity to aggregate may be a desirable or an undesirable characteristic that can be modulated by modifying the ligand. ${ }^{8}$ Numerous studies have been performed on large nanoparticles (diameter in $>100 \mathrm{~nm}$ ). In particular, large charged colloids interacting in electrolyte solutions have shown that they display attraction when equally charged. ${ }^{28-38}$ However, the theories developed for large colloids are not applicable to small nanoparticles. In fact, atomistic details are crucial in characterization of small AuNPs in aqueous solutions. ${ }^{15,18-19,39}$ Fortunately, with today's high performance computing facilities, it is feasible to simulate the stochastic dynamics of $10^{6}$ degrees of freedom of all the atoms constituting the AuNPs, the ligands, the waters, and the solute ions of an aqueous solution. ${ }^{13-19}$

In this article, we present a computational study of the interactions between small $\mathrm{Au}_{144}(\mathrm{SR})_{60}$ nanoparticles in an aqueous solution with eight combinatory concentrations of solute ions (tabulated in Table I). As shown in Fig. 1), each of these AuNPs is functionalized with 60 4-mercapto-benzoates, where the $\mathrm{R}$ group is $\mathrm{pMBA}^{-}$or its protonated form, pMBA. The AuNP has a core diameter of about $2 \mathrm{~nm}$ and its overall diameter (ligands included) is around $3.2 \mathrm{~nm}$. The solute ions are $\mathrm{Na}^{+}, \mathrm{K}^{+}, \mathrm{TMA}^{+}$(tetramethylamonium cation), $\mathrm{TRS}^{+}$ (trisamonium cation), $\mathrm{Cl}^{-}$, and $\mathrm{OH}^{-}$which are also illustrated in Fig. 1. Our objectives are to gain insights into how to modulate AuNP-AuNP interactions in an aqueous solution and to find optimal ways to reversibly concentrate AuNP solutions without precipitating irreversible aggregates of AuNPs.

\section{METHODS}

In essence, we simulate the stochastic dynamics of $\sim 10^{6}$ degrees of freedom of the constituent atoms of an aqueous solution. From the noisy dynamics data, we extract the thermodynamic functions that give definitive characteristics of a system's behavior, which include the potential of mean force, i.e., the reversible work, to bring two AuNPs to a given separation distance as well as the ion distribution densities around an AuNP. In the supporting information (SI), we give all the details of the procedures to build the model systems, to run the equilibrium molecular dynamics (MD), to run the nonequilibrium steered molecular dynamics (SMD) ${ }^{41}$ the work curves from the SMD runs, the formulas to compute the $\mathrm{PMF}^{42-47}$ from SMD work data, ${ }^{48-49}$ and the formulas to estimate the aggregation propensity from the PMF curves. ${ }^{19}$ Here, we briefly list the physical parameters used in this study and their sources. The initial structure of $\mathrm{Au}_{144} \mathrm{~S}_{60}$ core was taken from Refs. ${ }^{50-52}$. The interaction parameters for sulfur and gold (Au-Au and S-Au) are from Ref. ${ }^{53}$. All other interactions were represented by the CHARMM36 force field, ${ }^{54-55}$ to which we added the van der Waals (vdW) parameters for gold: $\sigma=1.66 \AA$ and $\varepsilon=-0.106 \mathrm{kcal} / \mathrm{mol}$. Water was represented with the TIP $3 \mathrm{P}^{56}$ model. The cut-off distance applied to the vdW interactions was $1.0 \mathrm{~nm}$, with a switching distance of $0.9 \mathrm{~nm}$ and a pair-list distance of $1.2 \mathrm{~nm}$. Langevin dynamics was implemented with a 1.0-fs time-step for short-range interactions and 4.0 fs for long-range interactions, and with a Langevin damping of $5.0 \mathrm{ps}^{-1}$. Temperature and pressure were maintained at $298 \mathrm{~K}$ and 1.0 bar. The model system dimensions are typically $10 \mathrm{~nm}$ along the $\mathrm{x}-$ and $\mathrm{y}$-axis and $20 \mathrm{~nm}$ along the $\mathrm{z}$-axis. Periodic boundary conditions were 
applied in all directions. Full electrostatic interactions were computed through the ParticleMesh Ewald (PME) method. All simulation runs were implemented with NAMD. ${ }^{57}$

\section{RESULTS AND DISCUSSION}

\section{Protonation of AuNPs}

In an aqueous solution, individual pMBA molecules, having a pKa of 4.2, are mostly deprotonated at $\mathrm{pH} 7$ or above. An AuNP having 60 pMBAs bonded on its surface in a monolayer, however, may have a different pKa value. ${ }^{39}$ While the uncertainty about the effective pKa of our AuNPs remains, it is certainly interesting to consider the case of AuNPs being half-way protonated, which is represented in our model System I. The characteristics of System I will be put in contrast with other systems of AuNPs being fully deprotonated. Specifically, System I is an aqueous solution containing $50 \mathrm{mM}$ of $\mathrm{Na}^{+}$and $1.67 \mathrm{mM}$ of $\mathrm{AuNP}^{-30}$. Each of the AuNPs is charged with -30e, equally distributed over the entire NP, with every other pMBA protonated, the remainder being deprotonated. The $\mathrm{Na}^{+}$cations neutralize the system overall.

Shown in Fig. 2, top panel, is the free-energy profile of System I where the PMF (or the reversible work to bring two AuNPs together) is plotted as a function of the separation distance between the centers of the two AuNPs. When the separation distance is greater than $3.4 \mathrm{~nm}$, the two AuNPs repel one another and the repulsive interaction strength is much lower than the direct Coulomb repulsion between two charges of $-30 \mathrm{e}$ each. This vastly reduced repulsive interaction can be readily expected as a result of the strong screening by an aqueous medium. Around the separation distance of $3.2 \mathrm{~nm}$, however, there is a PMF well of $4.2 \mathrm{kcal} / \mathrm{mol}$ in depth, indicating a metastable aggregation state at the short range when two AuNPs nearly touch one another. This effective attraction between two equally charged AuNPs actually has its root in the salt-bridge formed by a positively charged sodium ion $\mathrm{Na}^{+}$being attracted by a negatively charged $\mathrm{pMBA}^{-}$on one AuNP and, at the same time, being attracted by another negatively charged $\mathrm{pMBA}^{-}$on a second AuNP, ${ }^{19}$ which is illustrated in Fig. 3.

The AuNP propensity to aggregate depends on the strength of an individual salt-bridge and the number of salt-bridges in a given system, which are determined by the multiple factors of the system working concertedly on the overall level but stochastically on the atomic level. ${ }^{19}$ When one $\mathrm{Na}^{+}$falls in between two $\mathrm{PMBA}^{-}$groups, the energy of the system is lowered but the entropy is also reduced because the bridge-forming $\mathrm{Na}^{+}$loses its freedom to roam the entire aqueous volume. The probability for a salt-bridge to form is dependent upon the system's free-energy change caused by its formation. Therefore, the AuNP aggregation propensity can be sensitive to the tuning of any constituent factors of a system.

Also plotted in Fig. 2 (bottom panel) are the distribution densities of the ligand carboxyl groups as a function of the distance to the AuNP center and of the $\mathrm{Na}^{+}$ions as a function of the distance to the AuNP center. The carboxyl groups are distributed within a sharp peak around $1.4 \mathrm{~nm}$, indicating that the ligands are uniformly packed on the surface of the AuNP core of $144 \mathrm{Au}$ atoms and $60 \mathrm{~S}$ atoms which are bonded to each other rather rigidly and that the pMBAs are also quite rigid themselves. Integrating the area under the $\mathrm{Na}^{+}$density curve, 
we observe that approximately half of the $\mathrm{Na}^{+}$ions are in close contact with the AuNP surface, which are capable of forming salt-bridges. The other half diffuse around in the aqueous environment. The number of salt-bridges largely depends on the balance between the entropic drive for sodium ions to diffuse and the energetic drive for sodium ions to be trapped. This balance presents an efficient way to modulate the AuNP aggregation propensity by changing the sodium concentration of an aqueous solution of AuNPs.

It should be noted that the PMF (or reversible work) represents the overall, statistically averaged interaction between two nanoparticles. If we approximate it simply as the sum of all salt-bridges, we have a rough estimate of the salt-bridging strength by dividing the PMFattraction with the number of bridges we observe from the simulations. And the number of salt-bridges is directly related the surface curvature of the AuNP.

\section{Monoatomic ions vs near-spherical composite ions}

The characteristics of System II to VII are illustrated in Fig. 4. It should be noted that the AuNPs are all completely deprotonated in Systems II to VII. Each AuNP carries a total charge of $-60 \mathrm{e}$.

System II (having $1.67 \mathrm{mM}$ of AuNP -60 and $100 \mathrm{mM}$ of $\mathrm{Na}^{+}$) differs from System I only in the AuNP protonation state and the corresponding $\mathrm{Na}^{+}$concentration to keep the system neutral. The PMF of system II shown in Fig. 4(A) has a well near $3.1 \mathrm{~nm}$ that is $7 \mathrm{k}_{\mathrm{B}} \mathrm{T}$ below zero, indicating moderate AuNP aggregation propensity. Compared with System I, this stronger aggregation propensity comes from the fact that a higher concentration of charged carboxyl groups along with the correspondingly higher concentration of $\mathrm{Na}^{+}$ions leads to a greater number of salt-bridges formed between two AuNPs at a separation around $3.1 \mathrm{~nm}$.

Adding $100 \mathrm{mM}$ of $\mathrm{NaOH}$ to System II, we obtain System III whose characteristics are shown in Fig. 4(A) as well. The increased concentration of $\mathrm{Na}^{+}$leads to an even greater number of salt-bridges formed between a pair of AuNPs and thus a deeper PMF well. Examining the distribution densities of the ligand carboxyl groups, the cations, and the anions, we observe that $\mathrm{OH}^{-}$ions do not play a significant role other than neutralizing the system and that adjusting $\mathrm{Na}^{+}$concentration is a very effective way to modulate the aggregation propensity of AuNP aqueous solutions.

Replacing the $\mathrm{Na}^{+}$ions of Systems II and III with $\mathrm{K}^{+}$one to one, we obtain systems IV and $\mathrm{V}$, respectively. The characteristics of Systems IV and V resemble Systems II and III respectively. However, the PMF well of System IV $\left(-2 \mathrm{k}_{\mathrm{B}} \mathrm{T}\right)$ is not as deep as System II ($\left.7 \mathrm{k}_{\mathrm{B}} \mathrm{T}\right)$. Correspondingly, System IV has a weak propensity for AuNPs to aggregate. The PMF curve of System V is similar to that of System III but has a less deep well, which means moderate aggregation propensity. These similarities and dissimilar characteristics all stem from similar chemical properties between $\mathrm{Na}^{+}$and $\mathrm{K}^{+}$but different sizes of the two cations. The smaller $\mathrm{Na}^{+}$(diameter $2.7 \AA$ ) interacts with the ligand carboxyl groups more strongly than the larger $\mathrm{K}^{+}$(diameter $3.5 \AA$ ). Quantitative characterizations of these ionligand interactions in an aqueous environment are tabulated in the SI, Table S1. Therefore, the $\mathrm{Na}^{+}$salt-bridges between two AuNPs are stronger than the $\mathrm{K}^{+}$salt-bridges. Additionally, 
the space between a pair of AuNPs can accommodate fewer $\mathrm{K}^{+}$cations and thus a smaller number of salt-bridges, which is illustrated in the right panels of Figs. 4 (A) and (B). It is worth noting that the direct vdW attractions between two small AuNPs do not appear to play a significant role (shown in SI, Fig. S5) as they would in the case of large colloids and that the continuous medium approximations are not valid for small AuNPs. Atomistic details are essential in an accurate account of small AuNPs in aqueous solutions.

At this point, we conclude that the behavior of an AuNP aqueous solution is very sensitive to the size of the counterions (carrying charges opposite of the AuNP charge). It is a natural step forward to study aqueous solutions of AuNPs with $\mathrm{TMA}^{+}$as the counterions. $\mathrm{TMA}^{+}$ (diameter $4.6 \AA$ ), which carries a single charge and is tetrameric in symmetry, is not too dissimilar in shape from the perfectly spherical $\mathrm{Na}^{+} / \mathrm{K}^{+}$but is much more bulkier in volume. It displaces a larger number of waters in an aqueous solution and it is far less hydrophilic than $\mathrm{Na}^{+} / \mathrm{K}^{+}$. (The hydration energies of $\mathrm{TMA}^{+}$and $\mathrm{Na}^{+}$are shown in the SI, Fig. S6.) Consequently, it is quite ineffective to screen out the electric field of a charged AuNP (SI, Fig. S7). All these properties of $\mathrm{TMA}^{+}$together give rise to the very similar characteristics between System VI having $2.22 \mathrm{mM}$ of AuNP ${ }^{-60}$ and $133 \mathrm{mM}^{+} \mathrm{TMA}^{+}$and System VII having $2.22 \mathrm{mM}$ of AuNP ${ }^{-60}, 233 \mathrm{mM}$ of $\mathrm{TMA}^{+}$and $100 \mathrm{mM}$ of OH. Despite the $100 \mathrm{mM}$ difference in $\mathrm{TMA}^{+}$concentration, the PMF curves of Systems VI and VII (Fig. 4(C)) are essentially identical. In both systems, the Coulomb repulsion between a pair of AuNPs is not effectively screened out by the aqueous medium containing even high concentrations of $\mathrm{TMA}^{+}$counterions. The large size of $\mathrm{TMA}^{+}$and the symmetric distribution of its charge together make its interaction with the ligand carboxyl groups very weak (SI, Table S1) and the probability of forming salt-bridges very low (none observed in the simulations of this study). The AuNP aqueous solutions having only $\mathrm{TMA}^{+}$counterions exhibit no propensity to aggregate (in agreement with in vitro experiments ${ }^{58}$ ) and, at the meantime, a strong tendency against attaining a high concentration of AuNPs.

\section{Non-spherical composite ions}

Shown in Fig. 5 are the characteristics of System VIII that contains $150 \mathrm{mM}^{\circ}$ of TRS ${ }^{+}$and 50 $\mathrm{mM}$ of $\mathrm{Cl}^{-}$in addition to $1.67 \mathrm{mM}$ of AuNP ${ }^{-60}$. This aqueous solution of $\mathrm{AuNP}^{-60}$ has no propensity to aggregate but it differs distinctly from Systems VI and VII. Its PMF curve is essentially flat from $3.5 \mathrm{~nm}$ to a greater separation distance between two AuNPs whereas Systems VI and VII present strong repulsion in this range. System VIII (having TRS $^{+}$as counterions) has no propensity for aggregation and exhibits no repulsion between AuNPs beyond a separation distance of $3.5 \mathrm{~nm}$ in contrast with System VII.

Seeking the root of differences between System VI/VII and System VIII, we note that TRS ${ }^{+}$ differs from $\mathrm{TMA}^{+}$in every aspect other than both carrying a single positive charge $+\mathrm{e}$. $\mathrm{TRS}^{+}$is bulkier than $\mathrm{TMA}^{+}$by three hydroxyl groups (Fig. 1); $\mathrm{TRS}^{+}$is highly non-spherical in shape while $\mathrm{TMA}^{+}$is sterically tetrameric (Fig. 1); And $\mathrm{TRS}^{+}$is polar while $\mathrm{TMA}^{+}$is apolar. In fact, $\mathrm{TRS}^{+}$counterions interact strongly with the ligand carboxyl groups as demonstrated in the sharp peak of its distribution density curve (Fig. 5). Thus the TRS ${ }^{+}$ aqueous medium efficiently screens out the Coulomb repulsion between two negatively charged AuNPs so that the AuNPs can be easily brought together to form an aqueous 
solution of high AuNP concentration that can be reversibly diluted by adding a needed amount of $\mathrm{TRS}^{+}$aqueous solution. In supplemental information, Fig. S8, we plotted the PMF curves of Systems V to VIII $v$ s the PMF of a system of two fully charged AuNPs in pure water. From the contrast of the three curves, we are clear that $\mathrm{TMA}^{+}$ions neutralize the AuNPs partially and $\mathrm{TRS}^{+}$ions neutralize the AuNPs nearly completely.

It is interesting to note again that $\mathrm{TRS}^{+}$is distinctively different from spherical or nearspherical cations in its non-spherical shape and polarized charge distribution. Any other polar non-spherical composite ions should have similar functions when used as counterions in an AuNP aqueous solution. We therefore predict that it is feasible and effective to use non-spherical composite counterions in the production of reversibly highly concentrated AuNP aqueous solutions for medical and other applications.

\section{CONCLUSIONS}

Conducting in silico experiments of small AuNPs in an aqueous solution with eight different combinatory concentrations of monoatomic and composite ions: $\mathrm{Na}^{+}, \mathrm{K}^{+}, \mathrm{Cl}^{-}, \mathrm{TMA}^{+}$, $\mathrm{TRS}^{+}$, and $\mathrm{OH}^{-}$, we reached the following conclusions: (1) Small gold nanoparticles are indeed sensitive to changes in the aqueous environment, particularly, the size-and-shape and the concentration of the counterions. Ions with charge similar to AuNPs (both carrying negative charges in this paper) do not play a significant role other than neutralizing the total system. (2) Smaller counterions at higher concentrations cause a greater aggregation propensity of AuNPs. (3) Non-spherical composite counterions are very effective to eliminate the AuNP aggregation propensity and to screen out the direct Coulomb repulsion between the like-charged AuNPs. (4) Lowering the $\mathrm{pH}$ of an AuNP aqueous solution reduces the AuNP aggregation propensity even in the presence of sodium ions that are most efficient for salt-bridge formation. We speculate that these findings will be validated in in vitro experiments and will prove helpful in the design of AuNP based technologies.

\section{Supplementary Material}

Refer to Web version on PubMed Central for supplementary material.

\section{ACKNOWLEDGEMENTS}

The authors are grateful to Dr. Miguel Jose Yacaman for insightful discussions. They acknowledge support from the NIH (Grant \#GM084834) and the Welch Foundation (Project AX-1857). ODV also acknowledges support from the Mexican National Council of Science and Technology (CONACYT, grant \#533262). The computing time on supercomputers was provided by the Texas Advanced Computing Center.

\section{References}

1. Doane TL, Burda C. The Unique Role of Nanoparticles in Nanomedicine: Imaging, Drug Delivery and Therapy. Chem. Soc. Rev. 2012,; 41(7):2885-911. [PubMed: 22286540]

2. Leroueil PR, Berry SA, Duthie K, Han G, Rotello VM, McNerny DQ, Baker JR, Orr BG, Banaszak Holl MM. Wide Varieties of Cationic Nanoparticles Induce Defects in Supported Lipid Bilayers. Nano Lett. 2008,; 8(2):420-424. [PubMed: 18217783] 
3. Verma A, Uzun O, Hu Y, Hu Y, Han H-S, Watson N, Chen S, Irvine DJ, Stellacci F. SurfaceStructure-Regulated Cell-Membrane Penetration by Monolayer-Protected Nanoparticles. Nat Mater. 2008,; 7(7):588-595. [PubMed: 18500347]

4. Lin C-AJ, Yang T-Y, Lee C-H, Huang SH, Sperling RA, Zanella M, Li JK, Shen J-L, Wang H-H, Yeh H-I, et al. Synthesis, Characterization, and Bioconjugation of Fluorescent Gold Nanoclusters toward Biological Labeling Applications. ACS Nano. 2009,; 3(2):395-401. [PubMed: 19236077]

5. Cho EC, Xie J, Wurm PA, Xia Y. Understanding the Role of Surface Charges in Cellular Adsorption Versus Internalization by Selectively Removing Gold Nanoparticles on the Cell Surface with a I2/Ki Etchant. Nano Lett. 2009,; 9(3):1080-1084. [PubMed: 19199477]

6. Kim C, Agasti SS, Zhu Z, Isaacs L, Rotello VM. Recognition-Mediated Activation of Therapeutic Gold Nanoparticles inside Living Cells. Nat. Chem. 2010,; 2(11):962-966. [PubMed: 20966953]

7. Cho EC, Au L, Zhang Q, Xia Y. The Effects of Size, Shape, and Surface Functional Group of Gold Nanostructures on Their Adsorption and Internalization by Cells. Small. 2010,; 6(4):517-522. [PubMed: 20029850]

8. Murthy AK, Stover RJ, Borwankar AU, Nie GD, Gourisankar S, Truskett TM, Sokolov KV, Johnston KP. Equilibrium Gold Nanoclusters Quenched with Biodegradable Polymers. ACS Nano. 2012,; 7(1):239-251. [PubMed: 23230905]

9. Bartczak D, Muskens OL, Sanchez-Elsner T, Kanaras AG, Millar TM. Manipulation of in Vitro Angiogenesis Using Peptide-Coated Gold Nanoparticles. ACS Nano. 2013,; 7(6):5628-5636. [PubMed: 23713973]

10. McNamara K, Tofail SAM. Nanosystems: The Use of Nanoalloys, Metallic, Bimetallic, and Magnetic Nanoparticles in Biomedical Applications. Phys. Chem. Chem. Phys. 2015

11. Goeken KL, Subramaniam V, Gill R. Enhancing Spectral Shifts of Plasmon-Coupled Noble Metal Nanoparticles for Sensing Applications. Phys. Chem. Chem. Phys. 2015,; 17(1):422-427. [PubMed: 25406679]

12. Coelho SC, Rangel M, Pereira MC, Coelho MAN, Ivanova G. Structural Characterization of Functionalized Gold Nanoparticles for Drug Delivery in Cancer Therapy: A Nmr Based Approach. Phys. Chem. Chem. Phys. 2015,; 17(29):18971-18979. [PubMed: 26126833]

13. Lin J, Zhang H, Chen Z, Zheng Y. Penetration of Lipid Membranes by Gold Nanoparticles: Insights into Cellular Uptake, Cytotoxicity, and Their Relationship. ACS Nano. 2010,; 4(9):54215429. [PubMed: 20799717]

14. Lin J-Q, Zheng Y-G, Zhang H-W, Chen Z. A Simulation Study on Nanoscale Holes Generated by Gold Nanoparticles on Negative Lipid Bilayers. Langmuir. 2011,; 27(13):8323-8332. [PubMed: 21634406]

15. Heikkilä E, Gurtovenko AA, Martinez-Seara H, Häkkinen H, Vattulainen I, Akola J. Atomistic Simulations of Functional Au144(Sr)60 Gold Nanoparticles in Aqueous Environment. J. Phys. Chem. C. 2012,; 116(17):9805-9815.

16. Rocha, E. L. d.; Caramori, GF.; Rambo, CR. Nanoparticle Translocation through a Lipid Bilayer Tuned by Surface Chemistry. Phys. Chem. Chem. Phys. 2013,; 15(7):2282-2290. [PubMed: 23223270]

17. Heikkila E, Martinez-Seara H, Gurtovenko AA, Vattulainen I, Akola J. Atomistic Simulations of Anionic $\mathrm{Au}(\mathrm{Sr})$ Nanoparticles Interacting with Asymmetric Model Lipid Membranes. Biochim. Biophys. Acta. 2014,; 1838(11):2852-2860. [PubMed: 25109937]

18. Alsharif SA, Chen LY, Tlahuice-Flores A, Whetten RL, Yacaman MJ. Interaction between Functionalized Gold Nanoparticles in Physiological Saline. Phys. Chem. Chem. Phys. 2014,; 16(9):3909-3913. [PubMed: 24441708]

19. Villarreal OD, Chen LY, Whetten RL, Yacaman MJ. Ligand-Modulated Interactions between Charged Monolayer-Protected Au144(Sr)60 Gold Nanoparticles in Physiological Saline. Phys. Chem. Chem. Phys. 2015,; 17(5):3680-3688. [PubMed: 25556346]

20. Van Lehn RC, Alexander-Katz A. Ligand-Mediated Short-Range Attraction Drives Aggregation of Charged Monolayer-Protected Gold Nanoparticles. Langmuir. 2013,; 29(28):8788-8798. [PubMed: 23782293]

21. Ouyang R, Jiang D.-e. Ligand-Conformation Energy Landscape of Thiolate-Protected Gold Nanoclusters. J. Phys. Chem. C. 2015,; 119(37):21555-21560. 
22. Antosova A, Gazova Z, Fedunova D, Valusova E, Bystrenova E, Valle F, Daxnerova Z, Biscarini F, Antalik M. Anti-Amyloidogenic Activity of Glutathione-Covered Gold Nanoparticles. Mater. Sci. Eng. C. 2012,; 32(8):2529-2535.

23. Bowman M-C, Ballard TE, Ackerson CJ, Feldheim DL, Margolis DM, Melander C. Inhibition of Hiv Fusion with Multivalent Gold Nanoparticles. J. Am. Chem. Soc. 2008,; 130(22):6896-6897. [PubMed: 18473457]

24. Bresee J, Maier KE, Boncella AE, Melander C, Feldheim DL. Growth Inhibition of Staphylococcus Aureus by Mixed Monolayer Gold Nanoparticles. Small. 2011,; 7(14):2027-2031. [PubMed: 21630443]

25. Ackerson, CJ.; Powell, RD.; Hainfeld, JF. Chapter Nine - Site-Specific Biomolecule Labeling with Gold Clusters. In: Grant, JJ., editor. Methods in Enzymology. Vol. 481. Academic Press; 2010. p. 195-230.

26. Hainfeld JF, Liu W, Halsey CMR, Freimuth P, Powell RD. Ni-Nta-Gold Clusters Target HisTagged Proteins. J. Struct. Biol. 1999,; 127(2):185-198. [PubMed: 10527908]

27. Paillusson F, Dahirel V, Jardat M, Victor J-M, Barbi M. Effective Interaction between Charged Nanoparticles and DNA. Phys. Chem. Chem. Phys. 2011,; 13(27):12603-12613. [PubMed: 21670822]

28. Dahirel V, Jardat M. Effective Interactions between Charged Nanoparticles in Water: What Is Left from the Dlvo Theory? Curr. Opin. Colloid Interface Sci. 2010,; 15(1-2):2-7.

29. Ibarra-Armenta JG, Martin-Molina A, Quesada-Perez M. Influence of Monovalent Ion Size on Colloidal Forces Probed by Monte Carlo Simulations. Phys. Chem. Chem. Phys. 2011,; 13(29): 13349-13357. [PubMed: 21706120]

30. Jho YS, Safran SA, Pincus PA. Effect of Charge Inhomogeneity and Mobility on Colloid Aggregation. Langmuir. 2012,; 28(22):8329-8336. M. [PubMed: 22571282]

31. Manning GS. Counterion Condensation Theory of Attraction between Like Charges in the Absence of Multivalent Counterions. Eur. Phys. J. E. 2011,; 34(12):1-18.

32. Nagornyak E, Yoo H, Pollack GH. Mechanism of Attraction between Like-Charged Particles in Aqueous Solution. Soft Matter. 2009,; 5(20):3850-3857.

33. Netz RR, Orland H. Beyond Poisson-Boltzmann: Fluctuation Effects and Correlation Functions. Eur. Phys. J. E. 2000,; 1(2-3):203-214.

34. Tata BVR, Mohanty PS, Valsakumar MC. Bound Pairs: Direct Evidence for Long-Range Attraction between Like-Charged Colloids. Solid State Commun. 2008,; 147(9-10):360-365.

35. Turesson M, Jönsson B, Labbez C. Coarse-Graining Intermolecular Interactions in Dispersions of Highly Charged Colloids. Langmuir. 2012,; 28(11):4926-4930. [PubMed: 22404737]

36. Wu Y-Y, Wang F-H, Tan Z-J. Calculating Potential of Mean Force between Like-Charged Nanoparticles: A Comprehensive Study on Salt Effects. Phys. Lett. A. 2013,; 377(31-33):19111919.

37. Iglesias E, Prado-Gotor R. Interaction of Gold Nanoparticles Mediated by Captopril and SNitrosocaptopril: The Effect of Manganese Ions in Mild Acid Medium. Phys. Chem. Chem. Phys. 2015,; 17(1):644-654. [PubMed: 25407561]

38. Bohinc K, Lue L. The Interaction between Like-Charged Nanoparticles Mediated by Rod-Like Ions. J. Nanosci. Nanotechnol. 2015,; 15(5):3468-3475. [PubMed: 26504966]

39. Wang D, Nap RJ, Lagzi I, Kowalczyk B, Han S, Grzybowski BA, Szleifer I. How and Why Nanoparticle's Curvature Regulates the Apparent Pka of the Coating Ligands. J. Am. Chem. Soc. 2011,; 133(7):2192-2197. [PubMed: 21280574]

40. Humphrey W, Dalke A, Schulten K. Vmd: Visual Molecular Dynamics. J. Mol. Graphics. 1996; 14(1):33-38.

41. Chen LY. Exploring the Free-Energy Landscapes of Biological Systems with Steered Molecular Dynamics. Phys. Chem. Chem. Phys. 2011,; 13(13):6176-6183. [PubMed: 21359274]

42. Kirkwood JG. Statistical Mechanics of Fluid Mixtures. J. Chem. Phys. 1935,; 3(5):300-313.

43. Chandler D. Statistical Mechanics of Isomerization Dynamics in Liquids and the Transition State Approximation. J. Chem. Phys. 1978,; 68(6):2959-2970. 
44. Pratt LR, Hummer G, Garciá AE. Ion Pair Potentials-of-Mean-Force in Water. Biophys. Chem. 1994,; 51(2-3):147-165.

45. Roux B. The Calculation of the Potential of Mean Force Using Computer Simulations. Comput. Phys. Commun. 1995,; 91(1-3):275-282.

46. Allen TW, Andersen OS, Roux B. Molecular Dynamics - Potential of Mean Force Calculations as a Tool for Understanding Ion Permeation and Selectivity in Narrow Channels. Biophys. Chem. 2006,; 124(3):251-267. [PubMed: 16781050]

47. Wu J. Density Functional Theory for Chemical Engineering: From Capillarity to Soft Materials. AIChE J. 2006,; 52(3):1169-1193.

48. Chen LY. Nonequilibrium Fluctuation-Dissipation Theorem of Brownian Dynamics. J. Chem. Phys. 2008,; 129(14):144113-4. [PubMed: 19045140]

49. Chen LY, Bastien DA, Espejel HE. Determination of Equilibrium Free Energy from Nonequilibrium Work Measurements. Phys. Chem. Chem. Phys. 2010,; 12(25):6579-6582. [PubMed: 20463999]

50. Lopez-Acevedo O, Akola J, Whetten RL, Grönbeck H, Häkkinen H. Structure and Bonding in the Ubiquitous Icosahedral Metallic Gold Cluster Au144(Sr)60. J. Phys. Chem. C. 2009,; 113(13): 5035-5038.

51. Bahena D, Bhattarai N, Santiago U, Tlahuice A, Ponce A, Bach SBH, Yoon B, Whetten RL, Landman U, Jose-Yacaman M. Stem Electron Diffraction and High-Resolution Images Used in the Determination of the Crystal Structure of the Au144(Sr)60 Cluster. J. Phys. Chem. Lett. 2013,; 4(6):975-981. [PubMed: 23687562]

52. Tlahuice-Flores A, Black DM, Bach SBH, Jose-Yacaman M, Whetten RL. Structure \& Bonding of the Gold-Subhalide Cluster I-Au144cl60[Z]. Phys. Chem. Chem. Phys. 2013,; 15(44):1919119195. [PubMed: 24105400]

53. Hautman J, Klein ML. Simulation of a Monolayer of Alkyl Thiol Chains. J. Chem. Phys. 1989,; 91(8):4994-5001.

54. Brooks BR, Brooks CL, Mackerell AD, Nilsson L, Petrella RJ, Roux B, Won Y, Archontis G, Bartels C, Boresch S, et al. Charmm: The Biomolecular Simulation Program. J. Comput. Chem. 2009,; 30(10):1545-1614. [PubMed: 19444816]

55. Vanommeslaeghe K, Hatcher E, Acharya C, Kundu S, Zhong S, Shim J, Darian E, Guvench O, Lopes P, Vorobyov I, et al. Charmm General Force Field: A Force Field for Drug-Like Molecules Compatible with the Charmm All-Atom Additive Biological Force Fields. J. Comput. Chem. 2010,; 31(4):671-690. [PubMed: 19575467]

56. Jorgensen WL, Chandrasekhar J, Madura JD, Impey RW, Klein ML. Comparison of Simple Potential Functions for Simulating Liquid Water. J. Chem. Phys. 1983,; 79(2):926-935.

57. Phillips JC, Braun R, Wang W, Gumbart J, Tajkhorshid E, Villa E, Chipot C, Skeel RD, Kalé L, Schulten K. Scalable Molecular Dynamics with Namd. J. Comput. Chem. 2005,; 26(16):17811802. [PubMed: 16222654]

58. Laaksonen T, Ahonen P, Johans C, Kontturi K. Stability and Electrostatics of Mercaptoundecanoic Acid-Capped Gold Nanoparticles with Varying Counterion Size. Chemphyschem. 2006,; 7(10): 2143-9. [PubMed: 16969881] 


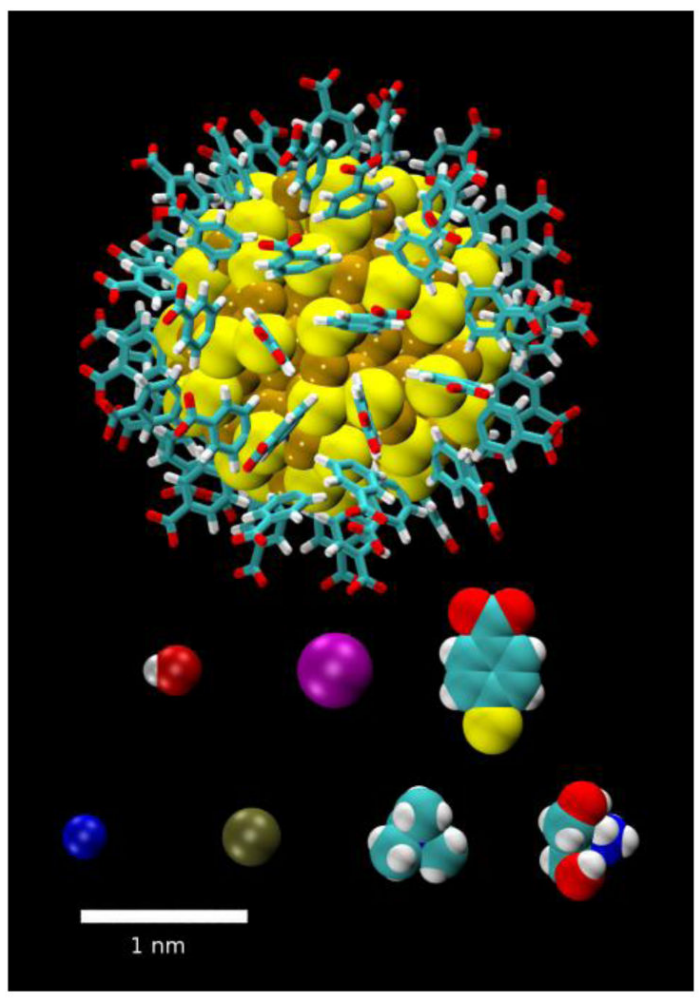

Fig. 1.

Equilibrium structures of the $\mathrm{Au}_{144}(\mathrm{SR})_{60}$ nanoparticle and other solutes shown on a common scale (bar length $=1.0 \mathrm{~nm}$ ). The ligand RS-groups are 4-mercapto-benzoates $\left[-\mathrm{SPh}\left(\mathrm{COO}^{-}\right)\left(\mathrm{pMBA}^{-}\right)\right]$. The effective diameter of the inorganic $\mathrm{Au}_{144} \mathrm{~S}_{60}$ core is $2.0 \mathrm{~nm}$. The $\mathrm{Au}$ and $\mathrm{S}$ atoms are represented as large spheres (van der Waals), and the ligands are in the licorice representation. The solute ions and the ligand are also shown as large spheres. In the middle row are (from left), respectively, $\mathrm{OH}^{-}, \mathrm{Cl}^{-}$, and $\mathrm{pMBA}^{-}$. In the bottom row are (from left), respectively, $\mathrm{Na}^{+}, \mathrm{K}^{+}, \mathrm{TMA}^{+}$, and $\mathrm{TRS}^{+}$. (Au: gold. S: yellow. O: red. C: cyan. $\mathrm{N}$ : blue. $\mathrm{H}$ : white. $\mathrm{Cl}$ : purple. Na: deep blue. $\mathrm{K}$ : tan.) All graphics in this paper were rendered with $\mathrm{VMD}^{40}$. 

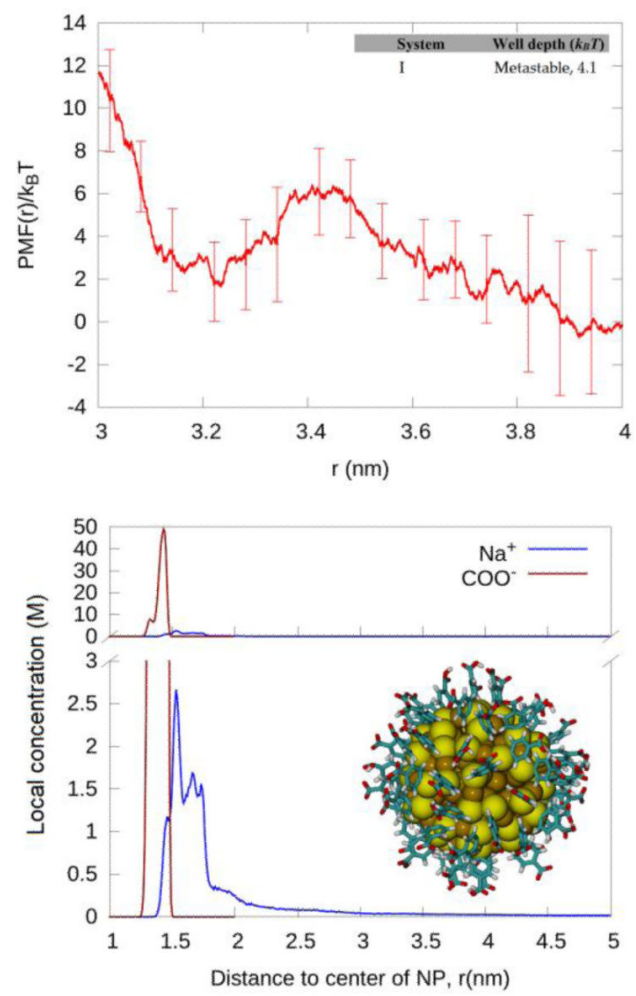

Fig. 2.

Characteristics of System I. Top: The PMF as a function of the center-to-center separation between two AuNPs. Bottom: The local concentrations (distribution densities) of $\mathrm{Na}^{+}$ions and the carboxyl groups around an AuNP. Inset in the bottom panel: A snapshot of AuNP in equilibrium aqueous solution. 


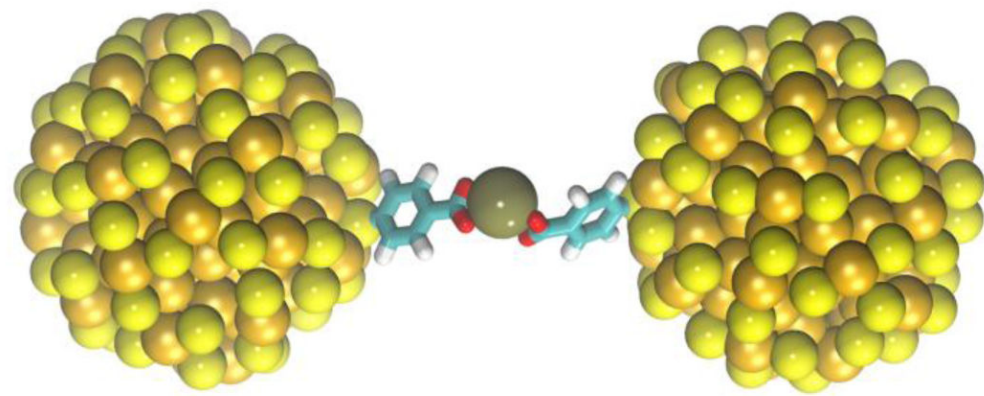

Fig. 3.

A salt-bridge formed by one $\mathrm{Na}^{+}$between two AuNPs. Only one ligand (pMBA) is shown on each AuNP. Na ${ }^{+}$ion (tan), $\mathrm{Au}$ (gold) and $\mathrm{S}$ (yellow) atoms are shown as large spheres. The ligands are shown as licorices $(\mathrm{H}$, white; $\mathrm{C}$, cyan; $\mathrm{O}$, red). 

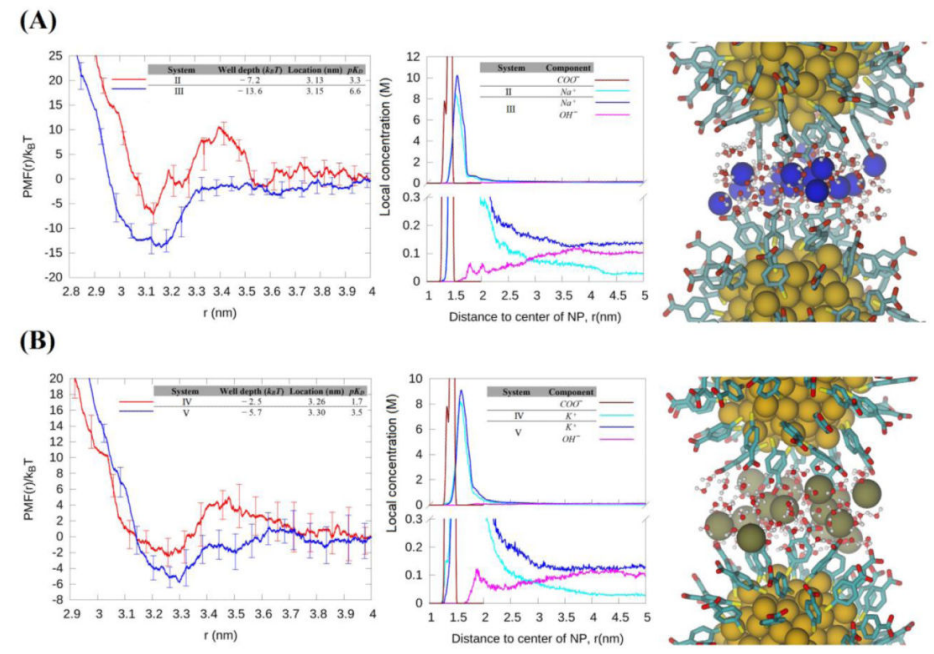

(C)

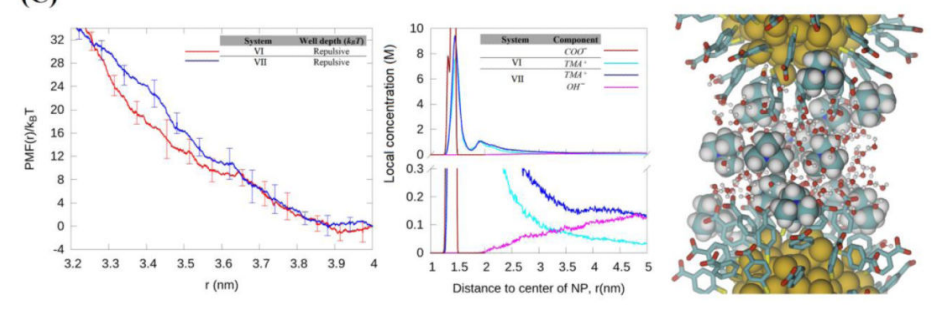

Fig. 4.

Characteristics of Systems II to VII. (A) Systems II and III. Left panel, the PMF as a function of the center-to-center distance between two AuNPs. Central panel, the distribution density of the ligand carboxyl groups and the same of the solute ions vs the distance to the AuNP center. Right panel, salt-bridges formed in System III. Here, $\mathrm{Na}^{+}$ions (blue) and the $\mathrm{Au}$ atoms (dark yellow) are represented by large spheres. The selected waters are in balland-sticks and the pMBAs are in licorice colored as follows: S, light yellow; C, cyan; O, red; H, white. (B) Systems IV and V. All identical to (A) except $\mathrm{K}^{+}$ions are in large tan spheres. (C) Systems VI and VII. All identical to (A) except that there are no salt-bridges in this case and $\mathrm{TMA}^{+}$ions are in large spheres. 

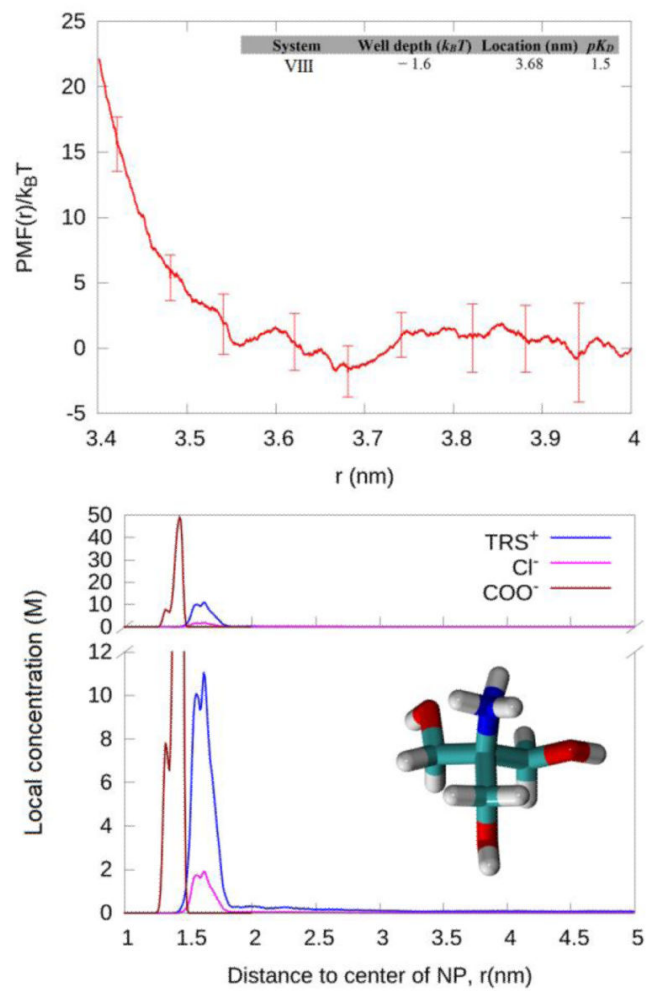

Fig. 5.

Characteristics of System VIII. Top: The PMF as a function of the center-to-center separation between two AuNPs. Bottom: The local concentrations of $\mathrm{TRS}^{+}, \mathrm{Cl}^{-}$, and the carboxyl groups around an AuNP. Inset in the bottom panel: A snapshot of $\mathrm{TRS}^{+}$in equilibrium aqueous solution represented in licorices colored as: $\mathrm{C}$, cyan; $\mathrm{H}$, white; $\mathrm{N}$, blue; and $\mathrm{O}$, red. 


\section{Table I}

All-atom model systems of AuNPs in an aqueous solution and their aggregation propensities.

\begin{tabular}{|l|l|l|}
\hline System & Solute concentrations $(\mathbf{m M})$ & Propensity \\
\hline I & {$\left[A u N P^{-30}\right]: 1.67 ;\left[\mathrm{Na}^{+}\right]: 50$.} & Very weak \\
\hline II & {$\left[\mathrm{AuNP}^{-60}\right]: 1.67 ;\left[\mathrm{Na}^{+}\right]: 100$.} & Moderate \\
\hline III & {$\left[\mathrm{AuNP}^{-60}\right]: 1.67 ;\left[\mathrm{Na}^{+}\right]: 200 ;\left[\mathrm{OH}^{-}\right]: 100$.} & Strong \\
\hline IV & {$\left[\mathrm{AuNP}^{-60}\right]: 2.22 ;\left[\mathrm{K}^{+}\right]: 133$.} & Weak \\
\hline V & {$\left[\mathrm{AuNP}^{-60}\right]: 2.22 ;\left[\mathrm{K}^{+}\right]: 233 ;\left[\mathrm{OH}^{-}\right]: 100$.} & Moderate \\
\hline VI & {$\left[\mathrm{AuNP}^{-60}\right]: 2.22 ;\left[\mathrm{TMA}^{+}\right]: 133$.} & None \\
\hline VII & {$\left[\mathrm{AuNP}^{-60}\right]: 2.22 ;\left[\mathrm{TMA}^{+}\right]: 233 ;\left[\mathrm{OH}^{-}\right]: 100$.} & None \\
\hline VIII & {$\left[\mathrm{AuNP}^{-60}\right]: 1.67 ;\left[\mathrm{TRS}^{+}\right]: 150 ;\left[\mathrm{Cl}^{-}\right]: 50$.} & None \\
\hline
\end{tabular}

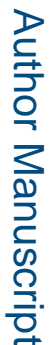

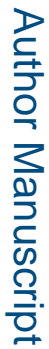

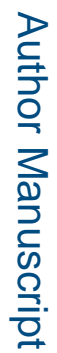

